\title{
Neural mass models as a tool to investigate neural dynamics during seizures
}

\author{
Tatiana Kameneva ${ }^{1, \#}$, Tianlin Ying ${ }^{1}$, Ben Guo ${ }^{1}$, Dean R. Freestone ${ }^{2,3}$ *
}

\begin{abstract}
Epilepsy is one of the most common neurological disorders and is characterized by recurrent seizures. We use theoretical neuroscience tools to study brain dynamics during seizures. We derive and simulate a computational model of a network of hippocampal neuronal populations. Each population within the network is based on a model that has been shown to replicate the electrophysiological dynamics observed during seizures. The results provide insights into possible mechanisms for seizure spread. We observe that epileptiform activity remains localized to a pathological region when a global connectivity parameter is less than a critical value. After establishing the critical value for seizure spread, we explored how to correct the effect by altering particular synaptic gains. The spreading of seizures is quantified using numerical methods for seizure detection. The results from this study provide a new avenue of exploration for seizure control.
\end{abstract}

Keywords: epilepsy, seizure spread, seizure suppression, signal processing, synaptic gain, EEG, neural mass model

\section{Introduction}

Epilepsy is a neurological disorder characterized by the recurrent seizures that are transient symptoms of synchronous neuronal activity in the brain (Fisher et al. 2005). Epilepsy is commonly diagnosed using electroencephalography (EEG), where abnormal electrical activities of the brain are captured. Epilepsy affects more than 50 million people worldwide. In the United States, it is estimated that 7 out of 1,000 people live with epilepsy (Hirtz et al. 2007). In Australia, over 225,000 people live with epilepsy and approximately $3 \%$ of Australians will experience epilepsy at some point in their lives

\footnotetext{
${ }^{* 1}$ Department of Electrical and Electronic Engineering, The University of Melbourne, Australia. ${ }^{2}$ Department of Statistics, Columbia University, New York, New York, USA. ${ }^{3}$ Department of Medicine, St. Vincent's Hospital, The University of Melbourne, Australia. \# corresponding author: tkam@unimelb.edu.au.
} 
(Australian Bureau of Statistics). The causes of epilepsy include trauma, central nervous system infections, tumors, perinatal and cerebrovascular disease (Fisher et al. 2005).

An understanding of the mechanisms underlying epileptic seizures is important for the development of the effective treatments. Studies have shown that in focal epilepsy ictogenesis may involve specific cortical and subcortical networks (Bartolomei et al. 2001), in temporal lobe epilepsy multiple foci could drive each other into a seizure (Bertman et al. 2009), and that in limbic epilepsy the synchrony dynamics across brain structures vary between seizure's initiation and termination phases (Sobayo et al. 2013). While experimental data has brought us closer to an understanding of the pathological brain dynamics, in many cases, mathematical modeling and computer simulations are the only possible venue to observe changes in a complex system and make solid predictions based on a comprehensive analysis.

Theoretical neuroscience involves the use of computational models to simulate the dynamics of neural populations and their interactions. Various mathematical and computational models have been developed to simulate neuronal dynamics. An overview of the dynamic brain models is discussed in (Deco et al. 2008). The neuronal models can be divided into single neuron models (Hodgkin and Huxley 1952) and neuronal networks models (Wilson and Cowan 1972, Fitzhugh 1955, Schelter et al. 2014). The last class can be divided into microscopic and macroscopic scale models. Detailed multicompartment microscopic models are often computationally intensive; therefore, macroscopic model are commonly utilized to study neuronal dynamics. These less complicated models can capture important brain dynamics such as alpha-like normal background brain activity and seizures.

Early macroscopic neural mass models (hereafter, simply, neural mass model) can be traced back to the 70s, when a lumped parameter model was proposed by Wilson and Cowan (1972) and Da Silva et al. (1974). Later, Jansen and Rit (1995) explored this model further and found the relationship between the excitatory and inhibitory neural populations that determine the kinetics of neural dynamics. Touboul et al. (2011) investigated the effect of parameter changes on the neural responses produced by Jansen and Rit's model, while Gribmert and Faugeras (2006) explored the behaviour of the model with respect to different external inputs. A modification of the Jansen and Rit's model was introduced by Wendling et al. by including fast inhibitory feedback in the network (Wendling et al. 2002). The advantages of the neural mass model include the physiological relevance of the model parameters, thus the results are helpful in the physiological interpretation of real data (Wendling et al. 2000). Furthermore, the parsimonious nature of neural mass models enables fitting to data, which may provide an opportunity for creating patient-specific descriptions of networks (Freestone et al. 2014a, Rowe et al. 2004).

Exploration of the effects of the brain network connectivity on the seizure spread was discussed in (Wang et al. 2014). We extended possible scenarios of the pathological brain dynamics discussed in (Wang et al. 2014) and investigated the network connectivity and 
intrinsic electrophysiology that could lead to seizure suppression. We proposed a hypothesis that a healthy brain network could have regions with pathological dynamics. However, this abnormal brain activity (including microseizures) is quickly suppressed by the strong influence of the healthy surrounding tissue. We explored necessary and sufficient conditions for seizure propagation and suppression. We proposed that having small regions of the brain with pathological dynamics is necessary but not sufficient condition for seizure propagation.

To verify this hypothesis, we extended Wendling's model to 16 interconnected neural columns joined in a hexagonal grid with torus boundary conditions. We explored seizure propagation and suppression in a network of interconnected columns under different physiological conditions. The effect of excitatory, slow and fast inhibitory gains and the strength of the connections between the network columns on seizure propagation and suppression were analyzed.

\section{Methods}

A computational model of a network of hippocampal neuronal populations was derived and simulated. Each population within the network was based on an existing model by Wendling et al. (2002) of intracranial EEG. Each node in the network model consisted of pyramidal, slow and fast inhibitory, and excitatory neural populations. A single column, seven interconnected columns, and sixteen interconnected columns were simulated and the dynamics were analyzed. The effect of the intrinsic electrophysiology of the columns and interconnection strengths in the networks on the seizure suppression and propagation were investigated. Seizure dynamics were quantified by calculating a feature, known as line-length, from the simulated EEG (Guo et al. 2010, Esteller et al. 2001) in combination with frequency and EEG offset values analysis. All simulations were run in Matlab with the sampling time step of $1 \mathrm{~ms}$.

\subsection{Single Column}

The model proposed by Wendling et al. (2002) was used to simulate intracranial EEG. In neural mass modelling, voltage dynamics of pyramidal neurons is often assumed to be representative of the recorded EEG signal. This convention is not electrophysiologically accurate. A schematic of the extracellular current flow is illustrated in Figure 4.a. Inhibitory connections to pyramidal cell's soma cause currents to pass across the cell's membrane and through the extracellular space, forming a source. Excitatory connections to pyramidal cell's distal dendrites form a current sink. At locations not too close to a neuron, the potential can be approximated by a current flow between two poles. Therefore, more appropriate approximation of the EEG signal is the size of extracellular dipole rather than mean firing rate of pyramidal cells (Nunez and Srinivasan 2006). 
To rectify this inaccuracy, we compared simulated output of a single column using three different mapping for EEG signal: standard neural mass model measurement function; summation of the absolute value of the incoming post-synaptic potentials to pyramidal populations; and summation of incoming firing rates to the pyramidal population from other populations within the network.

The model described interacting neural populations: pyramidal cells in the hippocampus or neocortex, slow-inhibitory interneurons, fast-inhibitory interneurons, and excitatory interneurons (spiny stellate cells). Slow inhibitory connections represented dendritic feedback to pyramidal cells and fast inhibitory connections represented somatic feedback to pyramidal cells. A single column represented average dynamics of approximately $10^{5}$ neurons and occupied approximately $1 \mathrm{~mm}^{2}$ of tissue.

A schematic diagram of a neural field model is illustrated in Figure 1. Units enclosing the 's' shape represent the action of the somas which is modeled by a sigmoidal activation function. The sigmoid converts a postsynaptic potential, $y_{i}, i=1,2, \ldots$, into an average spiking rate of a given population of cells. Small circles represent a second-order kernel that transforms an average population spike rate into an average postsynaptic potential (an output of a given population). Blue input to a column represents the input from distant brain areas (thalamus) and purple input to a column represents the input from neighboring cortical columns (set to zero for single column simulations). The influence of the distant brain areas into a column is represented by an excitatory input, $p(t)$, modeled as a Gaussian white noise. Numerical parameters used in simulations are given in Table 1 .

Similar to Wendling et al. (2002), a static sigmoidal function that converts a postsynaptic potential into an average spiking rate was described by

$$
S(y)=\frac{2 e_{0}}{1+\exp \left(r\left(y_{0}-y\right)\right)},
$$

where $y$ is a presynaptic mean membrane potential. The output of the model, $S(y)$, is the firing rate of a given population.

A post-synaptic response kernel was described by

$$
\begin{aligned}
& h_{e}(t)=A a \exp (-a t), \\
& h_{i}(t)=B b \exp (-b t), \\
& h_{g}(t)=G g \exp (-g t),
\end{aligned}
$$

where the subscript $e$ stands for the excitatory neural population, $i$ stands for the slowinhibitory population, and $g$ stands for the fast-inhibitory population, $t$ is time, $A, B, G$ are the average synaptic gains for a given population, the inverse of the parameters $a, b$ and $g$ are the dendritic average time constants in the feedback of a given population. $A, B$, and $G$ were varied. Examples of a response kernel and a sigmoid are shown in Figure 1.b. The membrane potential of pyramidal cells was assumed to be representative of the EEG signal recorded on surface electrodes, as illustrated in Figure 1.b. 
Table 1. Numerical parameters used in simulations.

\begin{tabular}{ll}
\hline Synaptic gains $A, B, G$ & varied in simulations \\
External excitatory input & $p(t)$, Gaussian White Noise \\
& $\mu=90 \mathrm{~Hz}, \sigma=30 \mathrm{~Hz}$ \\
Sigmoid parameters & $e_{0}=2.5 \mathrm{~s}^{-1}, r=0.56 \mathrm{mV}^{-1}, y_{0}=6 \mathrm{mV}$ \\
Response kernel parameters & $a=100 \mathrm{~s}^{-1}, b=50 \mathrm{~s}^{-1}, g=500 \mathrm{~s}^{-1}$ \\
Neurotransmitter depletion constant & $C=135$ \\
Synaptic constants & $C_{1}=C, C_{2}=0.8 C, C_{3}=C_{4}=0.25 C$ \\
& $C_{5}=0.3 C, C_{6}=0.1 C, C_{7}=0.8 C$
\end{tabular}

The coupling of four populations in one column depicted in Figure 1.b was described by a set of ten differential equations, repeated here from Wendling et al. (2002) for completeness:

$$
\left\{\begin{array}{l}
\dot{y}_{0}(t)=y_{5}(t) \\
\dot{y_{5}}(t)=A a S\left[y_{1}(t)-y_{2}(t)-y_{3}(t)\right]-2 a y_{5}(t)-a^{2} y_{0}(t) \\
\dot{y}_{1}(t)=y_{6}(t) \\
\dot{y}_{6}(t)=A a\left\{p(t)+C_{2} S\left[C_{1} y_{0}(t)\right]\right\}-2 a y_{6}(t)-a^{2} y_{1}(t) \\
\dot{y}_{2}(t)=y_{7}(t) \\
\dot{y}_{7}(t)=B b C_{4} S\left[C_{3} y_{0}(t)\right]-2 b y_{7}(t)-b^{2} y_{2}(t) \\
\dot{y_{3}}(t)=y_{8}(t) \\
\dot{y_{8}}(t)=G g C_{7} S\left[C_{5} y_{0}(t)-C_{6} y_{4}(t)\right]-2 g y_{8}(t)-g^{2} y_{3}(t) \\
\dot{y}_{4}(t)=y_{9}(t) \\
\dot{y}_{9}(t)=B b S\left[C_{3} y_{0}(t)\right]-2 b y_{9}(t)-b^{2} y_{4}(t) .
\end{array}\right.
$$

The values for the synaptic constants $C_{i}, i=1, \ldots, 7$, represented the average number of synaptic contacts between neural populations, were taken proportional to the parameter $C$ which accounted for a neurotransmitter depletion, see Table 1 for details. These numerical values were based on the experimental evidence, refer to (Jansen and Rit 1995, Wendling et al. 2002) for details.

The model described above produced six types of activity, including seizure-like:

Type 1: normal background activity;

Type 2: sporadic spikes;

Type 3: sustained discharge of spikes;

Type 4: slow rhythmic activity;

Type 5: low-voltage rapid activity;

Type 6: slow quasi-sinusoidal activity.

To enforce a certain types of activity onto a column, a non-unique set of parameters $A, B, G$ can be used. The values are given in the text for each simulation. The mathematical description of two interconnected columns and underlying equations are given in 
(Jansen and Rit 1995). The same mathematical framework can be extended to a large number of interconnected columns.

\subsection{Seizure Detection}

In order to quantify the dynamics of the network with a wide range of synaptic gains, a well-known feature of the EEG signal was used. To detect seizure-like activity, a linelength feature was used. The line-length feature is an efficient measure for seizure onset detection (Guo et al. 2010, Esteller et al. 2001). It is a measure of signal complexity that is sensitive to amplitude and frequency variations in the signal. The line-length was defined by

$$
L=\frac{1}{N-1} \sum_{t=1}^{N-1}|y(t+1)-y(t)|,
$$

where $\mathrm{N}$ is the number of samples in the experiment, $|\cdot|$ is the absolute value of the signal. The value of $L$ is dependent on the amplitude and frequency of the recorded signal. Therefore, the line-length is a good feature to classify the recorded EEG signal (output of the model) into six different types of activity. The line-length values in combination with frequency and EEG offset values were used to differentiate neural activity Types 1-6. To calculate the range of values of the line-length, frequency and EEG offset corresponding to Types 1-6, 100 simulations with different stochastic input noise $p(t)$ for each of the Types 1-6 with six different sets of $A, B, G$ were run, and minimum, maximum, and mean values were calculated. The parameters $A, B$, and $G$ used for these simulations are given in Table 2. 
Table 2. Parameters of the excitatory, slow and fast inhibitory gains $A, B$, and $G$ used to find the range of values for line-length, signal frequency and EEG offset to differentiate neural activity Types 1-6.

\begin{tabular}{|c|c|c|c|c|c|c|c|}
\hline & Parameters & Type 1 & Type 2 & Type 3 & Type 4 & Type 5 & Type 6 \\
\hline \multirow{3}{*}{ Set 1} & $\mathrm{~A}$ & 3.25 & 5.5 & 5.5 & 5.5 & 5.5 & 5.5 \\
\hline & B & 22 & 38 & 27 & 11 & 5 & 16 \\
\hline & $\mathrm{G}$ & 10 & 25 & 20 & 10 & 30 & 5 \\
\hline \multirow{3}{*}{ Set 2} & $\mathrm{~A}$ & 3.25 & 5.5 & 5.5 & 5.5 & 5.5 & 5.5 \\
\hline & B & 25 & 36 & 21 & 11 & 7 & 17 \\
\hline & $\mathrm{G}$ & 15 & 29 & 5 & 3 & 24 & 9 \\
\hline \multirow{3}{*}{ Set 3} & $\mathrm{~A}$ & 3.25 & 5.5 & 5.5 & 5.5 & 5.5 & 5.5 \\
\hline & B & 30 & 40 & 25 & 11 & 7 & 16 \\
\hline & G & 20 & 22 & 15 & 4 & 30 & 2 \\
\hline \multirow{3}{*}{ Set 4} & $\mathrm{~A}$ & 3.25 & 5.5 & 5.5 & 5.5 & 5.5 & 5.5 \\
\hline & B & 35 & 41 & 30 & 11 & 3 & 13 \\
\hline & G & 25 & 13 & 22 & 6 & 30 & 2 \\
\hline \multirow{3}{*}{ Set 5} & $\mathrm{~A}$ & 3.25 & 5.5 & 5.5 & 5.5 & 5.5 & 5.5 \\
\hline & $\mathrm{B}$ & 40 & 42 & 33 & 11 & 3 & 14 \\
\hline & G & 5 & 8 & 12 & 7 & 27 & 4 \\
\hline \multirow{3}{*}{ Set 6} & $\mathrm{~A}$ & 3.25 & 5.5 & 5.5 & 5.5 & 5.5 & 5.5 \\
\hline & B & 45 & 43 & 35 & 11 & 4 & 15 \\
\hline & $\mathrm{G}$ & 30 & 5 & 6 & 8 & 28 & 4 \\
\hline
\end{tabular}

\subsection{Seven Interconnected Columns: Intrinsic Electrophysiology}

To study spatial characteristics of the EEG signals, seven columns (a subset of the full network) were connected in a hexagonal structure and seizure propagation and suppression were analyzed under different physiological conditions. Seven interconnected columns is a generalization of a two-column network discussed in (Jansen and Rit, 1995).

A schematic diagram of a network of interconnected columns is illustrated in Figure 2.a. Black circle outlines and black arrows show the analyzed structure for seven columns. Parameters for pathological column 1 (red) were set to produce seizure-like activity Type 6: $A=5.5 \mathrm{mV}, B=16 \mathrm{mV}, G=20 \mathrm{mV}$. Parameters for healthy columns 2-7, surrounding the pathological column, were set to produce normal background activity Type 1: $A=5.5$ $\mathrm{mV}, B=16 \mathrm{mV}$, and $G=20 \mathrm{mV}$. All interconnections between columns were bidirectional. In the seven-column structure, $K_{1 j} \neq K_{i, 1}(i, j=2, \ldots, 7)$, (connections to/from the pathological columns are varied), while $K_{i j}=K_{i j}=15(i, j \neq 1)$, (connections between columns surrounding the pathological columns are the same in both directions). More precisely, the constant $K_{i j}$ is the connection strength from the population of neurons in the location $i$ to the population of neurons in the location $j$. The neural mass model is a phenomenological model and all parameter values should be taken as qualitative results.

The connectivity constant $K_{i j}$ can amplify $\left(K_{i j}>1\right)$ or attenuate $\left(K_{i j}<1\right)$ the output 
of the column $i$ before it is fed into the column $j$. The synaptic delay between columns is modelled by the delay kernel,

$$
h_{\mathrm{d}}(t)=A a_{\mathrm{d}} t e^{-a_{\mathrm{d}} t},
$$

for $t \geq 0$ and is set to zero for $t<0 ; a_{\mathrm{d}}=30 \mathrm{~s}^{-1}$.

\section{Seizure Propagation}

Due to the symmetric structure of the network around column 1, we limited our analysis of seizure propagation from pathological column 1 to healthy column 2. To explore seizure propagation, we systematically changed the strength of the forward connections, $K_{1 j}(j=2, \ldots, 7)$, from pathological column to healthy column. In addition, we explored seizure propagation when the excitatory synaptic gain, $A$, slow-inhibitory gain, $B$, and fast-inhibitory gain, $G$, were altered in column 2. To ensure that the seizure is propagated from column 1 to other columns and is not self-induced, we always ensured that the altered parameters $A, B, G$ were still producing background activity when the columns surrounding the pathological columns are disconnected from the network.

\section{Seizure Suppression}

To study the effect of the strength of the backwards connections (connections to the pathological column) on seizure suppression, we studied the dynamics of column 1 while varying backwards connections $K_{i 1}(i=2, \ldots, 7)$. All connectivity constants $K_{i 1}$ were changed simultaneously. Since the aim was to suppress the seizure activity in pathological column 1 by changing the excitability of the healthy columns and the intensity of the coupling strengths, all neighboring healthy columns 2-7 were used to reinforce the effect upon pathological column 1. Similar to the above, the excitatory synaptic gain $A$, slow-inhibitory gain $B$ and fast-inhibitory gain $G$ were altered in columns 2-7 (but still producing not-seizure-like activity) and the effect on seizure suppression was explored.

\subsection{Sixteen Interconnected Columns}

To study seizure propagation in a larger network, 16 columns were interconnected in the hexagonal-torus setup, as illustrated in Figure 2. This spatial structure allowed for wrapped-around boundary conditions that were more realistic for modeling a brain tissue by eliminating the effect of an arbitrary boundary. Parameters of one column in the network (shown by red in Figure 2.a were set to produce seizure-like activity Type 6, all other columns were set to produce normal background activity Type 1 . All connections between columns were bidirectional, $K_{i j}=K_{i j}$ (for all $i, j$ ). Two superimposed external inputs were fed to each column: Gaussian input noise, $p(t)$, and the direct superposition of the six outputs from the columns directly connected to the column receiving the input. The simulations explored the spread of seizures when the connectivity constants between columns, $K_{i j}$, were systematically altered. 


\section{Results}

Results demonstrated the conditions where seizure spread from the pathological to the neighboring healthy columns. Seizure remained local to the pathological column when the global connectivity parameter was less than a critical value. After establishing the critical value for seizure spread, we explored how to regulate individual columns to correct the effect of seizure spread. By locally reducing particular synaptic gains, the seizure propagation could be controlled.

\subsection{Single Column}

A single column disconnected from a network was able to produce six types of neural activity, as illustrated in Figure 3. Subplots (a) to (f) show activity Types 1 to 6 with parameters $A, B$, and $G$ for each type set as in (Wendling et al. 2002). Type 1 is normal background activity, all other types are seizure-like. The activity types are colorcoded for ease of referencing in the subsequent figures. While Types 2 and 3 had similar dynamics, the amplitude and frequency of the signal were different.

Output of a single column using three different mapping between membrane potential and EEG signal is illustrated in Figure 4.b-g. For simulations, parameters were set according to (Wending et al. 2002) to generate normal background activity (subplots b-d) or epileptic activity Type 3 (subplots e-g). Standard neural mass model measurement function is illustrated in subplots b and e, summation of the absolute value of the incoming post-synaptic potentials to pyramidal populations is shown is subplots c and f; and summation of incoming firing rates to the pyramidal population from other populations within the network is depicted in subplots $d$ and g. Results show that different mapping of EEG signal lead to similar results. Results show that despite the fact that the neural mass model is phenomenological and lacks accurate biophysical interpretation, it can be appropriately used to approximate EEG signal.

\subsection{Seizure Detection}

A method that uses a line-length and spectral feature analysis was used to quantify EEG changes. To explore the line-length values for Types 1 - 6 produced by the model, 100 samples for each type with different stochastic input noise, $p(t)$, were simulated and the line-length was recorded for each simulation. The range of obtained line-length values was used for the activity type detection in further simulations. The range of the line-length values for each neural activity Types 1-6 are given in Table 3 . 
Table 3. The range of obtained line-length values for Types 1-6.

(100 samples for each type were simulated)

\begin{tabular}{lllllll}
\hline & Type 1 & Type 2 & Type 3 & Type 4 & Type 5 & Type 6 \\
\hline max & 0.0014 & 0.2275 & 0.2651 & 0.0028 & 0.0026 & 0.3084 \\
mean & 0.0013 & 0.2178 & 0.2447 & 0.0021 & 0.0017 & 0.2752 \\
min & 0.0012 & 0.2085 & 0.2254 & 0.0017 & 0.0013 & 0.2026 \\
std & 0.0000 & 0.0074 & 0.0159 & 0.0002 & 0.0003 & 0.0375 \\
2std above & 0.0013 & 0.2325 & 0.2765 & 0.0024 & 0.0024 & 0.3503 \\
2std below & 0.0013 & 0.2031 & 0.2129 & 0.0018 & 0.0010 & 0.2001 \\
\hline
\end{tabular}

The results showed that the line-length values lie in the overlapped between regions for different activity types. Therefore, the line-length value (L), in combination with frequency (F) and EEG offset (D) values were used to differentiate neural activity types. A decision tree algorithm to quantify and detect the EEG types is shown in Figure 5, where the values of $L, D, F$ were taken from range of values obtained for Types 1-6: $L=0.1=(2$ std above Type $4+2$ std below Type 6$) / 2, D=4.98=(2$ std above Type $1+2$ std below Type 4$) / 2, F=15.36=(2$ std above Type $4+2$ std below Type 5$) / 2, F=7.08=(2$ std above Type $2+2$ std below Type 6$) / 2, F=4.15=2$ std below Type 3 .

\subsection{Seven Interconnected Columns: Intrinsic Electrophysiology}

\section{Seizure Propagation}

Figure 6 illustrates the effect of changing synaptic gains in column 2 on seizure propagation under three different scenarios. The output of the healthy column 2 is categorized into six types, which have been introduced in Methods. The horizontal axis, $K_{21}$, represents the backwards connectivity strength from healthy column 2 to pathological column 1 , the vertical axis, $K_{12}$, represents the forward connectivity strength from the pathological to the healthy column. The same color scheme for Types $1-6$ is used as in Figure 3. Color bar indicates the type of the activity, where Type 1 is normal background activity (dark blue), all other types are seizure-like activity. Results showed that the likelihood of seizure propagation to a neighboring healthy column is increasing as $K_{12}$ increases. This is intuitive, since the impact of one column onto another is dependent on the coupling strength and the larger the strength is, the stronger the effect. Note, the backwards connection $K_{21}$ did not affect the output dynamics of healthy column 2 .

Figures 6.a illustrates the effect of the excitatory synaptic gain $A$ in healthy column 2 on the seizure propagation from the pathological column. Results showed that with the increase of the synaptic gain, the propagation of seizure happened at a lower value of the connectivity constant $K_{12}$. In other words, it was easier for seizure to propagate in a hyper-excited (while still healthy) strongly connected network.

The effects of the slow inhibitory synaptic gain $B$ and fast inhibitory synaptic gain $G$ in the healthy column on seizure propagation are illustrated in Figures 6.b and 6.c, 
respectively. Results showed minimal effect of the fast inhibition, $G$, in the healthy column on seizure propagation, while the effect of the slow inhibition, $B$, was more noticeable.

The insert in Figure 6.c illustrates the simulated network with pathological column 1 in the centre (red) surrounded by healthy columns. The insert shows an example of seizure propagation for the following simulation parameters: Column 1: $A=5.5 \mathrm{mV}$, $B=16 \mathrm{mV}, G=5 \mathrm{mV}$; Column 2: $A=3.3 \mathrm{mV}, B=22 \mathrm{mV}, G=30 \mathrm{mV}$; Columns 3-7: $A=5.5 \mathrm{mV}, B=50 \mathrm{mV}, G=20 \mathrm{mV}$. For this example, the pathological column has slow quasi-sinusoidal activity (red, similar to Figure 3). This seizure-type activity propagates to healthy column 2 and this column now has sustained discharge of spikes (Type 3 activity, blue, similar to Figure 3).

To explore seizure propagation in detail, the output of column 2 when the connectivity strengths between columns 1 and 2 were altered is plotted in Figures 7.a,b. Figure 7.a depicts results when the forward connectivity $K_{12}$ is increased, while the backward connectivity $K_{21}$ is set to a constant value $\left(K_{21}=15 \mathrm{in}\right.$ (a) and $\left.K_{21}=50 \mathrm{in}(\mathrm{b})\right)$. Results showed that with the increasing strength of the $K_{12}$ connection, the column's output amplitude and frequency change from the normal background activity to the seizure-like activity. Figure 7 illustrates EEG dynamics when the forward connectivity $K_{12}$ is increased, while the backwards connectivity $K_{21}$ is constant. Results show that for $K_{12}>25$ the output of column 2 changes from normal background activity to seizure-like activity.

\section{Seizure Suppression}

Figure 8 depicts seizure suppression when the excitability of the neighboring healthy columns change. Intuitively, one would expect that increasing the excitability of the neighboring columns, would lead to an easier seizure propagation but not seizure suppression. However, by increasing inter-connectivity of the healthy neighboring columns (but still in the range of the healthy normal background activity), the positive feedback (connections from the healthy columns to the pathological column) is increased and the influence of the healthy columns on the pathological column becomes greater. This lead to seizure suppression as shown in Figure 8. The output of pathological column 1 is shown for different connection strengths from the healthy to the pathological column and for different excitatory synaptic gains $A$ in the healthy column. Note how the seizure observed in column 2 for $K_{21}<60$ and $A=3$ is suppressed for $K_{21} \geq 30$ and $A=7$. Note, this suppression does not lead to seizure-like dynamics in the other columns since there is no seizure-like activity to propagate through the network.

To explore seizure propagation in detail, the output of pathological column 1 when the connectivity strengths between columns 1 and 2 are altered for different values of the excitatory and slow inhibitory gains in column 2, are plotted in Figures 9.a,b,c. Top: changes in $K_{12}$ and $K_{21}$ that correspond to the output of column 1 below. Middle: magnitude of the signal output of column 1. Bottom: frequency of the signal output of column 1. $K_{1 j}=K_{12}, K_{i 1}=K_{21}(i, j=3, \ldots, 7), K_{i j}=15(i, j \neq 1)$. Simulation parameters - Column 1: $A=5.5 \mathrm{mV}, B=16 \mathrm{mV}, G=5 \mathrm{mV}$; Columns 2-7: $A=5 \mathrm{mV}$, $B=5 \mathrm{mV}, G=5 \mathrm{mV}$. b) Columns 2-7: $A=10 \mathrm{mV}, B=5 \mathrm{mV}, G=5 \mathrm{mV}$. c) $A=10$ 
$\mathrm{mV}, B=45 \mathrm{mV}, G=5 \mathrm{mV}$. Figures 9.a,b illustrate that a seizure can be suppressed for large values $K_{21}$, i.e. decrease in the amplitude and frequency of signal. Note the different excitatory gain $A$ in the subplots (a) and (b). However, when the slow inhibitory gain, $B$, in column 2 is increased (the column becomes less excited and therefore has less influence of pathological column 1), the magnitude of the signal does not change much and the signal's frequency is increased (the seizure is not suppressed), as depicted in Figure 9.c.

\subsection{Sixteen Interconnected Columns: Torus Structure}

To explore the spread of seizure in the 16-column torus structure, the connectivity constants between columns, $K_{i j}(i, j=1, \ldots, 16)$, were systematically varied. Initially, a large range of connectivity constants values were explored, ranging from 0 to 310 with a step size of 10. Results depicted in Figure 10 showed that for strong connectivity constants, $K_{i j} \geq 20$, seizures propagated in the network. $K_{i j}=0$ (disconnected columns) trivially lead to the only pathological column producing seizure-like activity. In Figure 10, the values of the connectivity constants $K_{i j}$ used in simulations are shown above the subplot, each subplot represent a network of 16 columns shown by 16 circles. In simulations, $K_{i j}=K_{j i}$ (for all $i, j$ ). To explore the propagation of seizure in details, the simulations were repeated with a smaller step size of 0.32 for $K_{i j}$ from 20 to 29.92. Results of these simulations are shown in Figure 10.b. The results showed non-trivial column transitions from the normal background activity to seizure-like dynamics Types 3, 4 and 6.

\section{Conclusions and Discussions}

This study highlights the importance of network dynamics in studying epilepsy. Previous computational studies have highlighted how pathological changes in an isolated foci lead to seizure-like dynamics. Here we show that the pathological changes that have previously been described do not necessary lead to seizures. A broad context must be taken into account when interpreting previous results when studying isolated brain regions. The key result of our simulations is that epileptic dynamics from an isolated pathology can be quenched or drowned by neighboring brain regions. Therefore, discovery of pathological brain tissue is not sufficient evidence to identify an epileptogenic zone. On the contrary, it is possible to have pathological tissue and normal neural dynamics.

The findings raise the question, what is the key aspect of epilepsy. There is evidence that non-epileptic people have constrained micro-seizures that do not clinically manifest as epilepsy (Stead et al. 2010). It is unknown what percentage of people have constrained micro-seizures, but given the small number of subjects in studies where micro-seizures have been investigated and the high detection rates, it is likely that the occurrence is high. Therefore, we speculate that the network topology plays a crucial, and perhaps defining role in governing seizure dynamics, separating epileptic and non-epileptic cases. 
Furthermore, correlations between localized pathologies and seizures are not uniquely causative mechanisms.

In this study, we used theoretical neuroscience approach to investigate brain dynamics during seizures. We used computer simulations of the interacting neural populations, to analyze physiological conditions that lead to seizure spread and suppression. Our results showed that there is a critical value for the connectivity strength of the network above which the seizure spreads. This spread can be regulated by increasing the influence of the healthy columns surrounding the pathological tissue. Our results are supported by multielectrode recordings showing that healthy brain have a potential to have selfcontained micro-seizures (Stead et al. 2010) and by the experimental evidence of an inhibitory restraint of seizure activity (Schevron et al. 2012). The results from our study provide insights into the process of seizure spread and generalization. These insights may provide a new avenue of exploration for seizure control. Future work will involve a larger exploration of the parameter space and an expansion of the model to include more columns.

The results of such studies can provide greater insights into how seizure could be contained by implantable bionic devices. By understanding the brain dynamics of individual patients, it is possible to tailor therapeutic neurostimulation to individual patients in the most optimal way. Electrical stimulation of neural tissue has been used to restore function to visually impaired people and people with hearing loss, for treatment of epilepsy and Parkinson's disease and psychiatric disorders (Ahuja et al. 2010, Clark 2003, Han et al. 2014, Holtzheimer and Mayberg 2011). The tools developed here can be used to investigate the effect of electrical stimulation on pathological tissues in patients with Parkinson's disease, to explore connectivity of the network for patients with psychiatric disorders, and, in general, to understand neural dynamics of a pathological tissue and to propose potential therapies.

\section{Acknowledgments}

This research was supported by the Australian Research Council through the Discovery Early Career Researcher Award (DECRA) DE120102210. Dr Freestone acknowledges the support of the Australian-American Fulbright Association. Dr Freestone would also like to thank Prof Liam Paninski at Columbia University for support and guidance. The authors would also like to thank and acknowledge important input from Prof Mark Cook, Dr Andre Peterson, Prof David Grayden, Prof Anthony Burkitt and all the members of the NeuroEngineering lab at The University of Melbourne. 


\section{References}

Ahuja, A.K., Dorn, J.D., Caspi, A., McMahon, M.J., Dagnelie, G.,da Cruz, L., Stanga, P., Humayun, M.S., and Greenberg, R.J. (2010) Blind subjects implanted with the Argus II retinal prosthesis are able to improve performance in a spatialmotor task. Br. J. Ophthalmol. 95(4): 539 - 543.

Australian Bureau of Statistics. http://www.abs.gov.au.

Bartolomei, F., Wendling, F., Bellanger, J.J., Regis, J., and Chauvel, P. (2001) Neural networks involving the medial temporal structures in temporal lobe epilepsy. Clin. Neurophysiol., 112: 1746 - 1760.

Bertman, E.H. (2009) Temporal lobe epilepsy: Where do the seizures really begin? Epilepsy Behav., 14: 32 - 37.

Clark, G. (2003) Cochlear Implants: Fundamentals and pplications. Springer Vergas New York Inc.

Da Silva, D.F., Hoeks, A., Smits, H., and Zetterberg, L.H. (1974) Model of brain rhythmic activity. Kybernetik, 15(1): $27-37$.

Deco, G., Jirka, V.K., Robinson, P.A., Breakspear, M., and Friston, K. (2008) The dynamic brain: from spiking neurons to neural masses and cortical fields. PLoS Comput. Biol., 4(8): $1-35$.

Esteller, R., Echauz, J., Tcheng, T., and Litt, B. (2001) Line length: an efficient feature for seizure onset detection. Proc. IEEE EMBS conf., 1707 - 1710.

Fisher, R.S., van Emde Boas, W., Blume, B., Elger, C., Genton, P., Lee, P., and Engel, Jr. J.(2005) Epileptic seizures and epilepsy: Definitions proposed by the international league against epilepsy (ILAE) and the international bureau for epilepsy (IBE). Epilepsia, 46: 470 - 474.

Salanova, V., Witt T., Worth R., Henry T.R., Gross R.E., Nazzaro J.M., Labar D., Sperling M.R., Sharan A., Sandok E., Handforth A., Stern J.M., Chung S., Henderson J.M., French J., Baltuch G., Rosenfeld W.E., Garcia P., Barbaro N.M., Fountain N.B., Elias W.J., Goodman R.R., Pollard J.R., Troster A.I., Irwin C.P., Lambrecht K., Graves N., Fisher R. SANTE Study Group (2015) Long-term efficacy and safety of thalamic stimulation for drug-resistant partial epilepsy. J. Neurology, 84(10): 1017 - 1025.

FitzHugh, R. (1955) Mathematical models of threshold phenomena in the nerve membrane. Bulletin Math. Biophys., 17(4): 257 - 278. 
Freestone, D.R., Aramd, P., Dewarc, M., Scerrif, K., Grayden, D.B., and Kadirkamanathand, V. (2011) A data-driven framework for neural field modeling. NeuroImage, 56(3): $1043-1058$.

Grimbert F., and Faugeras, O. (2006) Bifurcation analysis of jansen's neural mass model. Neural Comput., 18(12): 3052 - 3068.

Guo, L., Rivero, D., Dorado, J., Rabunal, J.R., Pazos, A. (2010) Automatic epileptic seizure detection in EEGs based on line length feature and artificial neural networks. J Neurosci. Methods, 191(1): 101 - 109.

Han, C.L., Hu, W., Stead, M., Zhang, T., Zhang, J.G., Worrell, G.A., and Meng, F.G. (2014) Electrical stimulation of hippocampus for the treatment of refractory temporal lobe epilepsy. Brain Res. Bull., 109: 13-21.

Hirtz, D., Thurman, D., Gwinn-Hardy, K., Mohammad, M., Chaudhuri, A.R., Zalutsky, R. (2007) How common are the "common" neurologic disorders? Neurology, 68(5): $326-337$.

Holtzheimer, P.E., and Mayberg, H.S. (2011) Deep brain stimulation for psychiatric disorders, Annu. Rev. Neurosci., 34: 289 - 307.

Hodgkin, A.L., and Huxley, A.F. (1952) A quantitative description of membrane current and its application to conduction and excitation in nerve. J. Physiol., 117(4): $500-544$.

Jansen, B.H., and Rit, V.G. (1995) Electroencephalogram and visual evoked potential generation in a mathematical model of coupled cortical columns. Biolog. Cybern., 4: 357 - 366 .

Jou, S.B., Kao, I.F., Yi, P.L., and Chang F.C. (2014) Electrical stimulation of left anterior thalamic nucleus with high-frequency and low-intensity currents reduces the rate of pilocarpine-induced epilepsy in rats. Europ. J. Epilepsy, 22(3): 221 230 .

Nunez, P.L., Srinivasan, R. (2006) Electric fields of the brain. Oxford University Press, Second Edition, Oxford New York.

Rowe, D.L., Robinson, P.A., Rennie C.J., Harris, A.W., Felmingham, K.L., Lazzaro, I.L., and Gordon, E. (2004) Neurophysiologically-based mean-field modelling of tonic cortical activity in post-traumatic stress disorder (PTSD), schizophrenia, first episode schizophrenia and attention deficit hyperactivity disorder (ADHD). $J$. Integrat. Neurosci, 3(4): 453 - 487. 
Schelter, B., Mader, M., Mader, W., Sommerlade, L., Platt, B., Lai, Y.C., Gregory, C., and Tiel, M. (2014) Overarching framework for data-based modelling. Front. Phys., 105: 30004. item[] Schevron, C.A., Weiss, S.A., McKhann Jr, G., Goodman, R.R., Yuste, R., Emerson, R.G., Trevelyan, A.J. (2012) Evidence of an inhibitory restraint of seizure activity in humans. Nature Communications, doi: $10.1038 /$ ncomms 2056 .

Sobayo, T., Fine, A.S., Gunnar, E., Kazlauskas, C., Nicholls, D., and Mogul, D.J. (2013) Synchrony dynamics across brain structures in limbic epilepsy vary between initiation and termination phases of seizures. IEEE Trans. Biomed. Eng., 60(3): $821-829$.

Stead, M., Bower, M., Brinkmann, B.H., Lee, K., Marsh W.R., Meyer, F.B., Litt, B., van Gompel, J., and Worrell, G.A. (2010) Microseizures and the spatiotemporal scales of human partial epilepsy. Brain, 133: 2789 - 2797.

Touboul, J., Wendling, F., Chauvel, P., and Faugeras, O. (2011) Neural mass activity, bifurcations, and epilepsy. Neural Comput., 23(12): 3232 - 3286.

Wang, Y., Goodfellow, M., Taylor, P.N., and Baier, G. (2014) Dynamic Mechanisms of Neocortical Focal Seizure Onset. PLoS Comput. Biol., 10(8): e1003787.

Wendling, F., Bartolomei, F., Bellanger, J.J., and Chauvel, P. (2002) Epileptic fast activity can be explained by a model of impaired GABAergic dendritic inhibition. Europ. J. Neurosci., 9: 1499 - 1508.

Wendling, F., Bellanger, J.J. Bartolomei, F., and Chauvel, P. (2000) Relevance of nonlinear lumped-parameter models in the analysis of depth-EEG epileptic signals. Biol. Cybern., 4: 367 - 378.

Wilson H.R., and Cowan, J.D. (1972) Excitatory and inhibitory interactions in localized populations of model neurons. Biophys. J., 12(1): 1 - 24. 

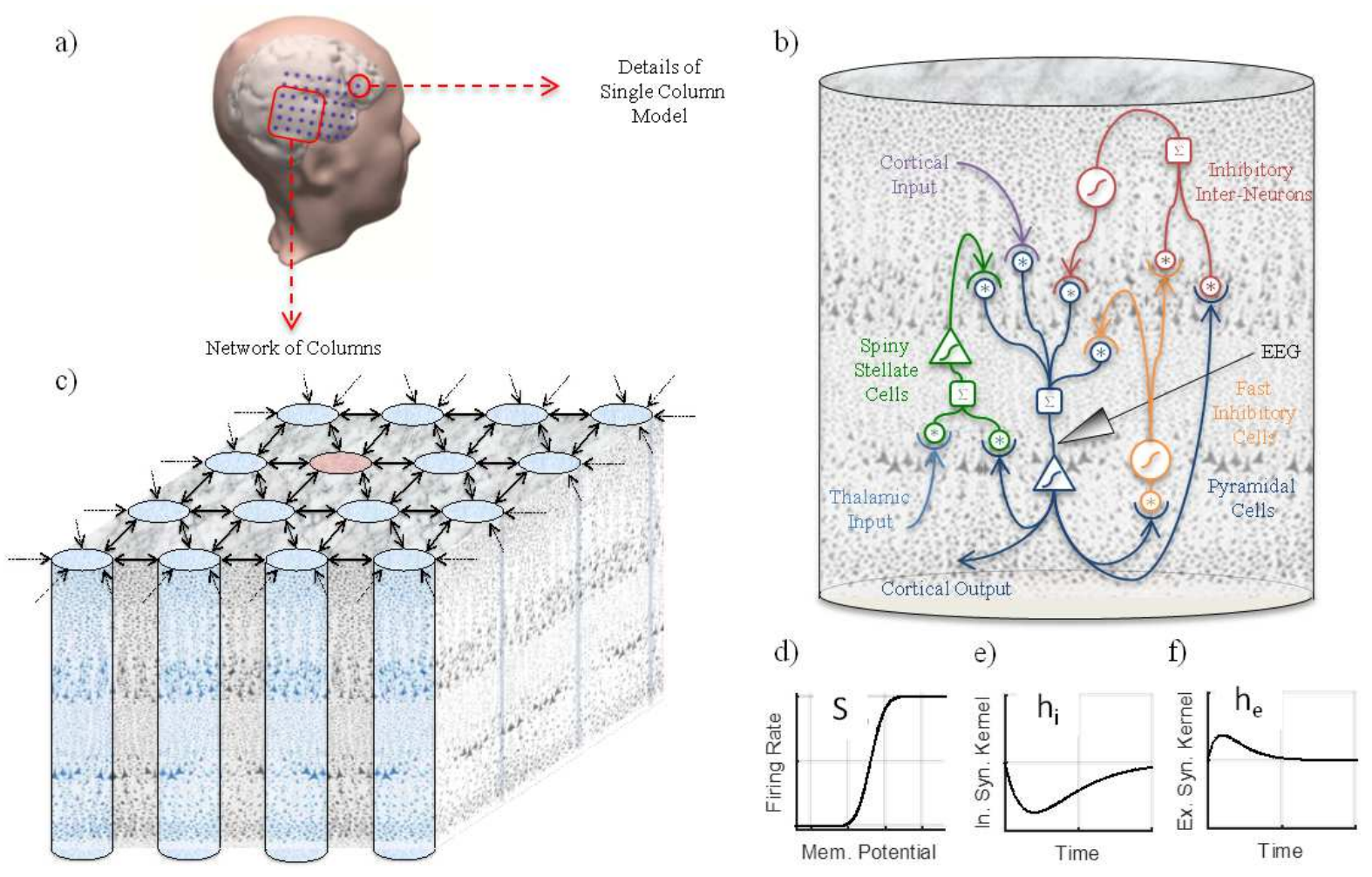

Fig. 1: A schematic diagram of a neural field model. a) An example of a column structure representing a population of $10^{5}$ neurons and an example of a network of 16 columns representing a larger brain tissue dynamics. An electrode array is shown by solid blue dots. b) A column of neurons, representing interactions of pyramidal neurons, slow and fast inhibitory interneurons, and excitatory spiny stellate cells. Large circles and a triangles: a static nonlinear function that converts a postsynaptic potential, $y_{i}, i=1,2, \ldots$, into an average spiking rate of a given populations. Small circles: a second order kernel that transforms an average population spike rate into an average postsynaptic potential. Input into the column from distant brain areas (thalamus), modelled as Gaussian noise. c) A network of 16 interconnected columns. The neuronal activity of pyramidal cells is interpreted as recorded EEG signal. d)-f) Examples of sigmoidal nonlinear function, inhibitory and excitatory response kernels. 

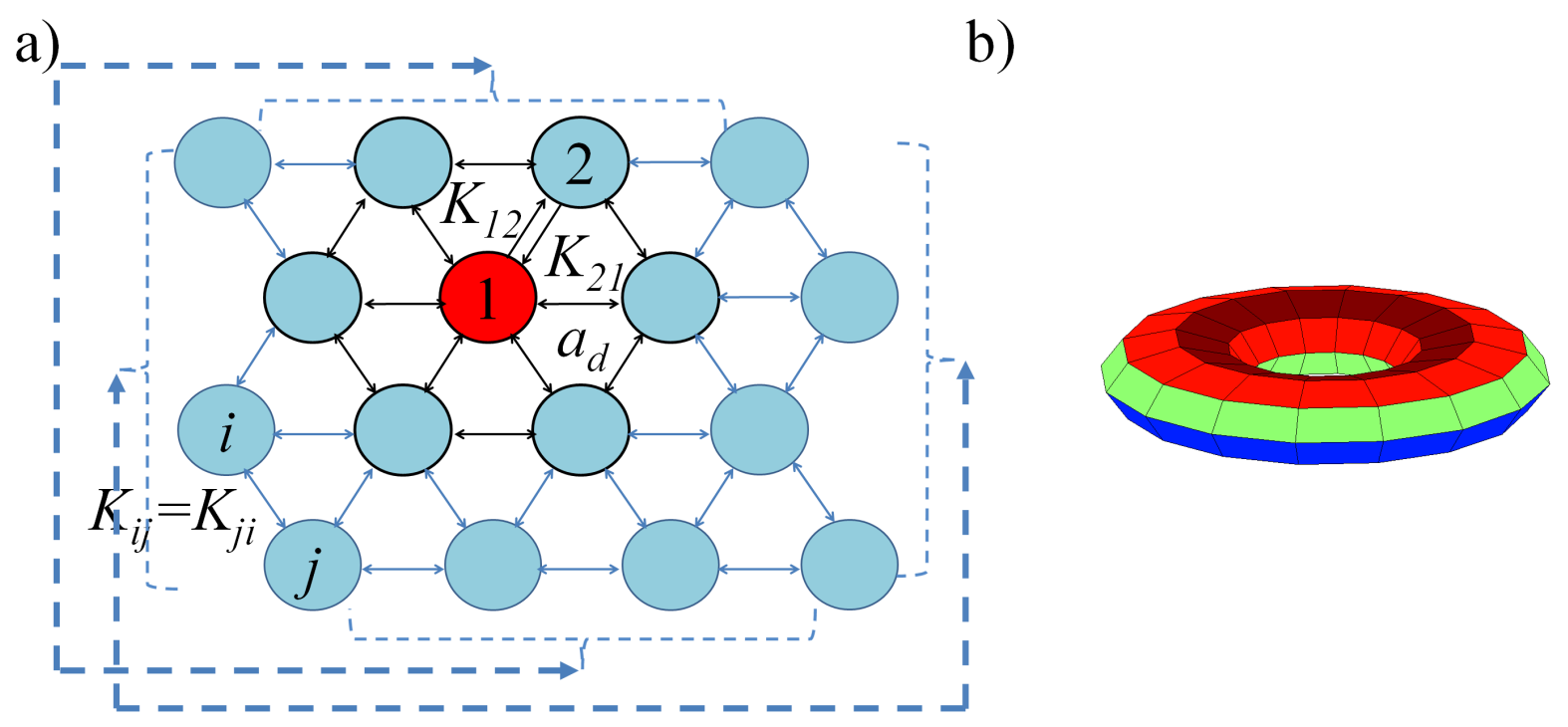

Fig. 2: A schematic diagram of a network of interconnected columns of neural populations. Parameters for red column are set to produce seizure-like activity. Parameters for all other columns are set to produce normal background activity when disconnected from the network. All interconnections between columns are bidirectional. Connectivity delay $a_{\mathrm{d}}$ and connectivity constants $K_{i j}$ are shown for some connections. a) Network of 16 interconnected columns. Black circle outlines and black arrows show the analyzed structure for seven columns. In the sevencolumn network, $K_{1 j} \neq K_{i 1}(i, j=2, \ldots, 7)$ and $K_{i j}=K_{i j}(i, j \neq 1)$. In the 16-column network, $K_{i j}=K_{j i}$ for all $i, j$ (connections are equal in both directions). Connections between boundaries are shown by dashed arrows (wrapped around). The excitatory input $p(t)$ is different for each column, not shown here for clarity. b) A torus structure used to analyze the dynamics of 16 interconnected columns. 
a) Normal background activity

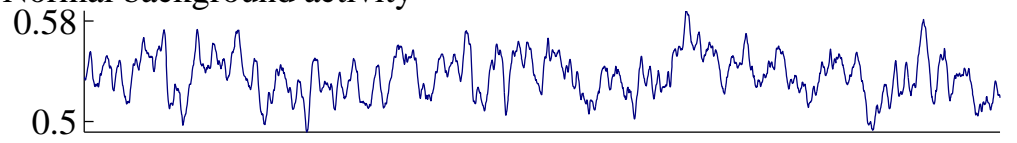

b) Sporadic spikes

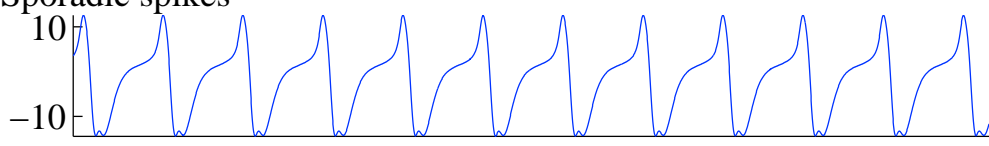

c) Sustained discharge of spikes

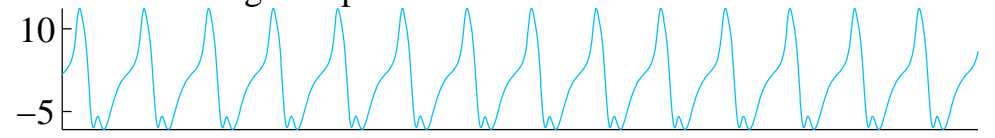

d) Slow rhythmic activity

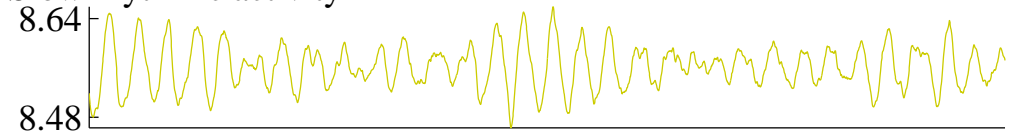

e) Low-voltage rapid activity

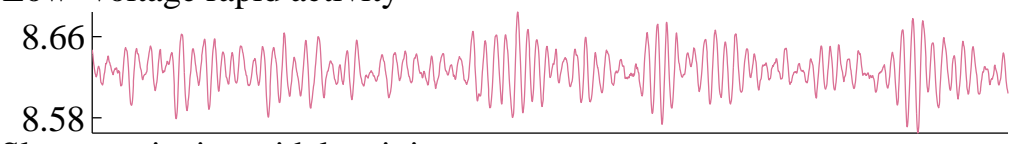

f) Slow quasi-sinusoidal activity

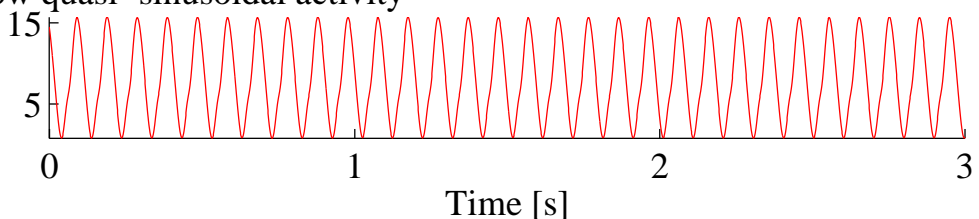

Fig. 3: Six different types of activity produced by a single column disconnected from the network. The model output represents the average postsynaptic potential of pyramidal cells. a) Type 1: normal background activity; b) Type 2: sporadic spikes; c) Type 3: sustained discharge of spikes; c) Type 4: slow rhythmic activity; d) Type 5: low-voltage rapid activity; e) Type 6: slow quasi-sinusoidal activity. $A, B$, and $G$ are set the same as in (Wendling et al. 2002). 


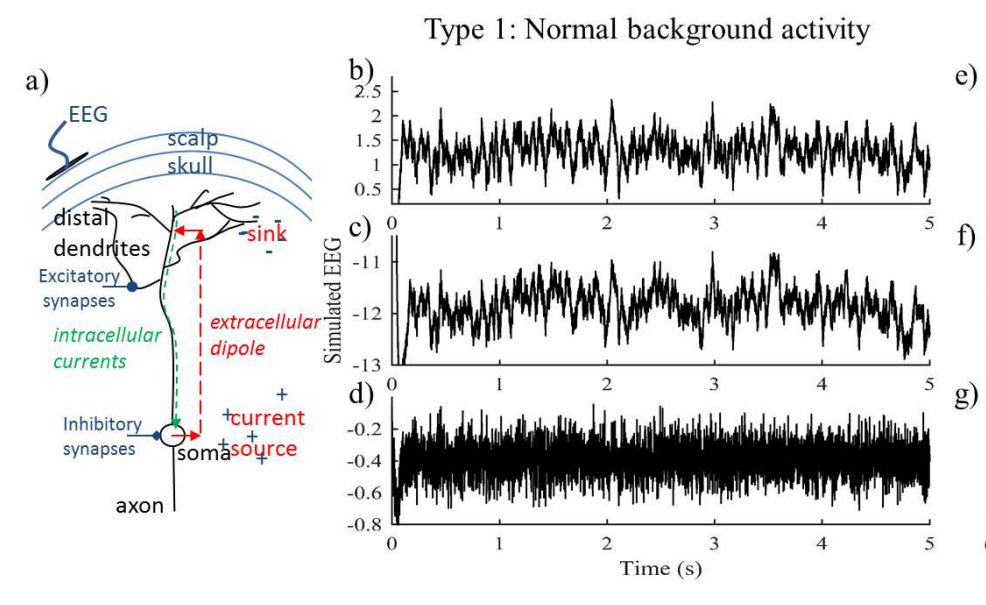

Type 3: Sustained discharge of spikes

e)
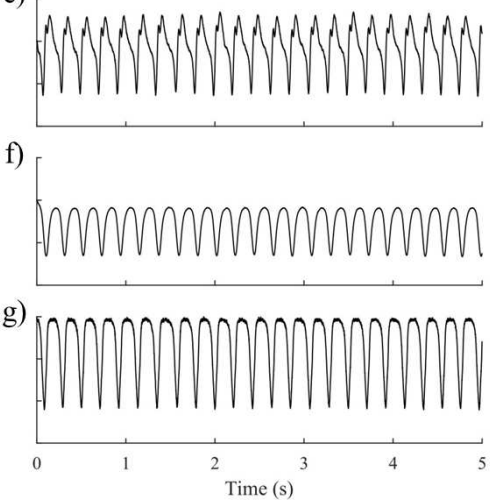

Fig. 4: a) The effect of synaptic activity on a single neuron. The EEG signal is approximated as a size of a dipole formed by synaptic currents. b)-g) Examples of various measurement functions. b),e) Standard neural mass model measurement function. c),f) Summation of the absolute value of the incoming post-synaptic potentials to pyramidal population. d),g) Summation of incoming firing rates to the pyramidal population from other populations within the network. For simulations, parameters were set according to Wending et al., (2002) to generate normal background activity (subplots b-d) or epileptic activity (subplots e-g).

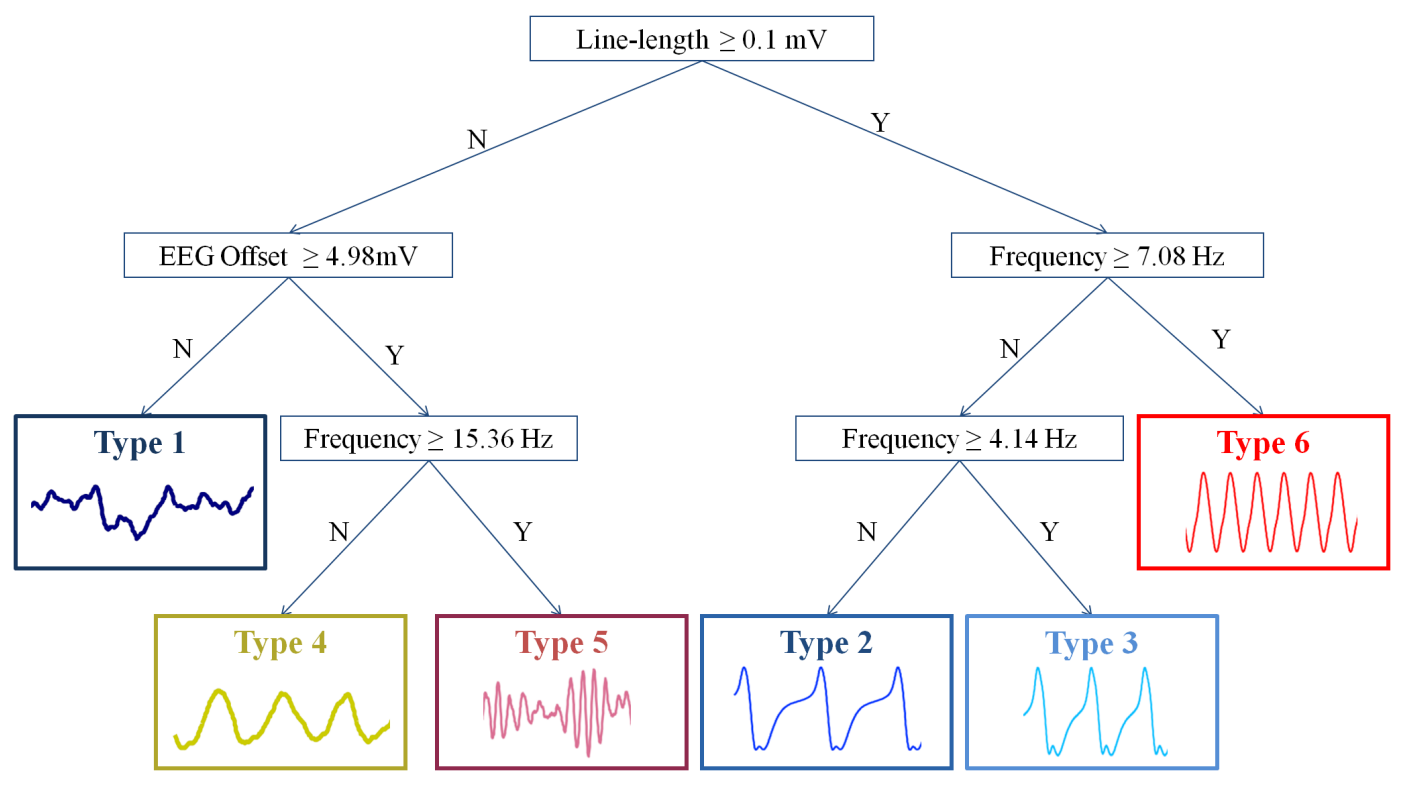

Fig. 5: The decision tree used to classify activity types based on frequency, line-length value and DC offset of the simulated signal. ' $\mathrm{Y}$ ' corresponds to the condition satisfied, 'N' corresponds to the condition not satisfied. The color-coded activity Types 1-6 are the same as types illustrated in Fig. 3. 
$\mathrm{A}=4.2$

$A=4.4$

$A=4.6$

$A=4.8$
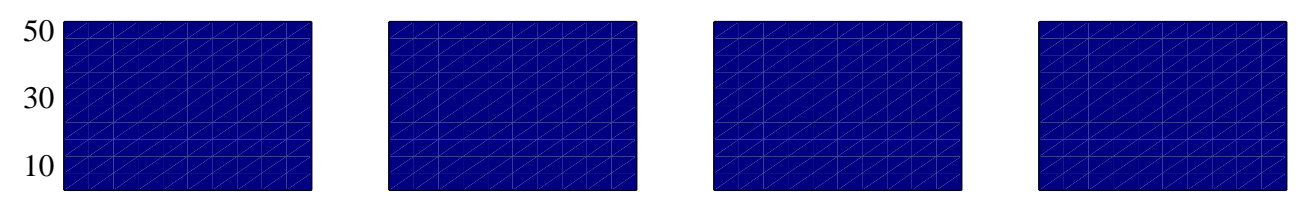

$\mathrm{A}=5.0$

$\mathrm{A}=5.2$

$\mathrm{A}=5.4$

$\mathrm{A}=5.6$
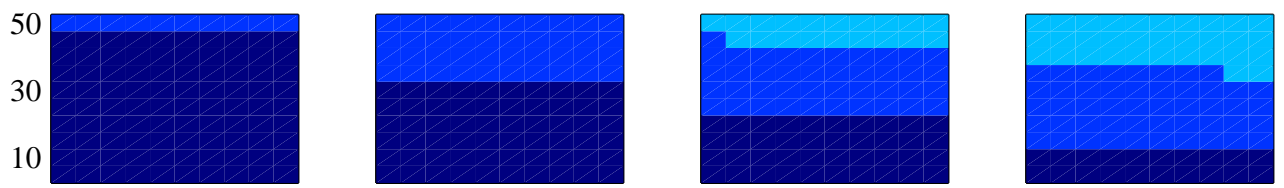

$\mathrm{A}=5.8$

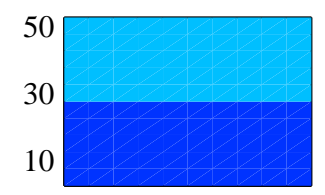

$\mathrm{A}=6.0$

b) Effect of slow inhibitory gain $B$

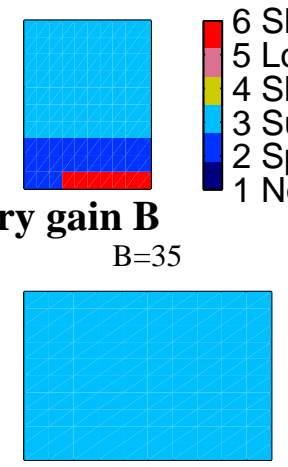

Slow quasi-sinusoidal

5 Low voltage rapid

4 Slow rythmic

3 Sustained discharge

2 Sporadic spikes

Normal background

$\mathrm{B}=30$

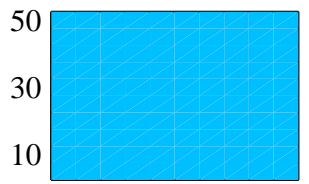

$\mathrm{B}=50$

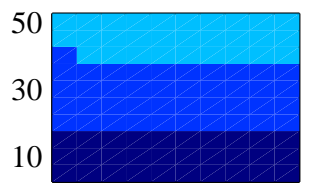

c) Effect of fast inhibitory gain $\mathbf{G}$

$\mathrm{G}=5$

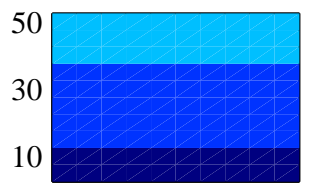

$\mathrm{G}=25$

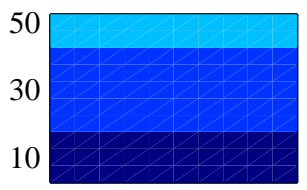

$\mathrm{G}=45$

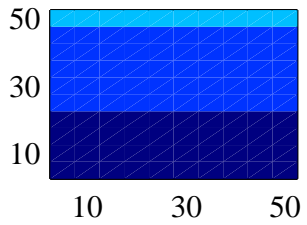

$\mathrm{B}=55$

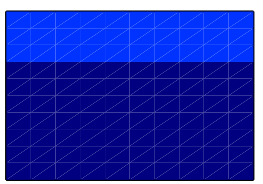

$\mathrm{G}=10$

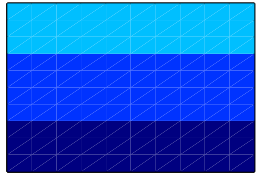

$\mathrm{G}=30$

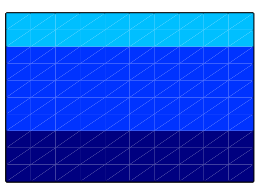

$\mathrm{G}=50$

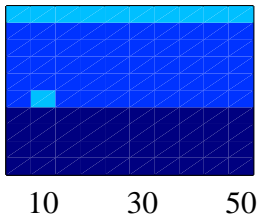

$\mathrm{B}=40$

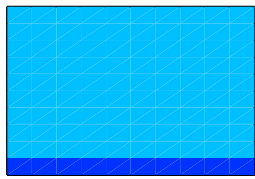

Con-ty from col1

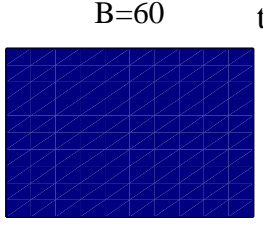

$\mathrm{G}=15$

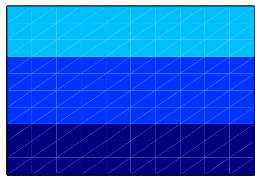

$\mathrm{G}=35$

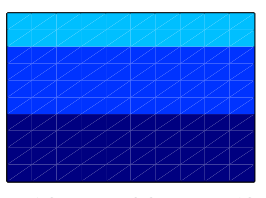

$10 \quad 30 \quad 50$
$B=45$

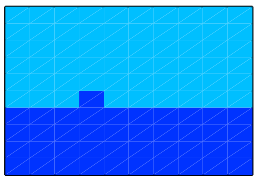

to $\operatorname{col} 2, \mathrm{~K} 12$

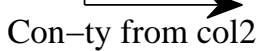

to col1, K21

$\mathrm{G}=20$

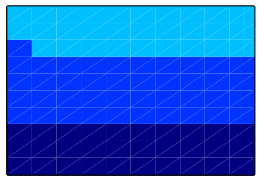

$\mathrm{G}=40$

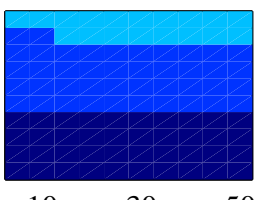

$\begin{array}{lll}10 & 30 & 50\end{array}$

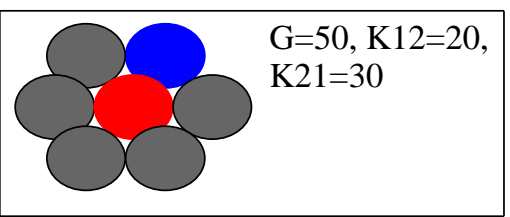

Fig. 6: Output of healthy column 2. The effect of the excitatory synaptic gain $A$, slow inhibitory gain $B$, and fast inhibitory gain $G$ in healthy column 2 on seizure propagation from column 1 to column 2. The type of activity in pathological column 1 is set to Type 6 (slow quasi-sinusoidal). Column 1: $A=5.5 \mathrm{mV}, B=16 \mathrm{mV}, G=5 \mathrm{mV}$; Columns 3-7: $A=5.5 \mathrm{mV}, B=50 \mathrm{mV}, G=20$. $K_{i j}=15(i, j \neq 1)$. Column 2 : a) $A$ is varied, $B=50 \mathrm{mV}, G=20$. b) $A=5.5 \mathrm{mV}, B$ is varied, $G=20$. c) $A=5.5 \mathrm{mV}, B=16 \mathrm{mV}, G$ is varied. Insert: see text for details. 

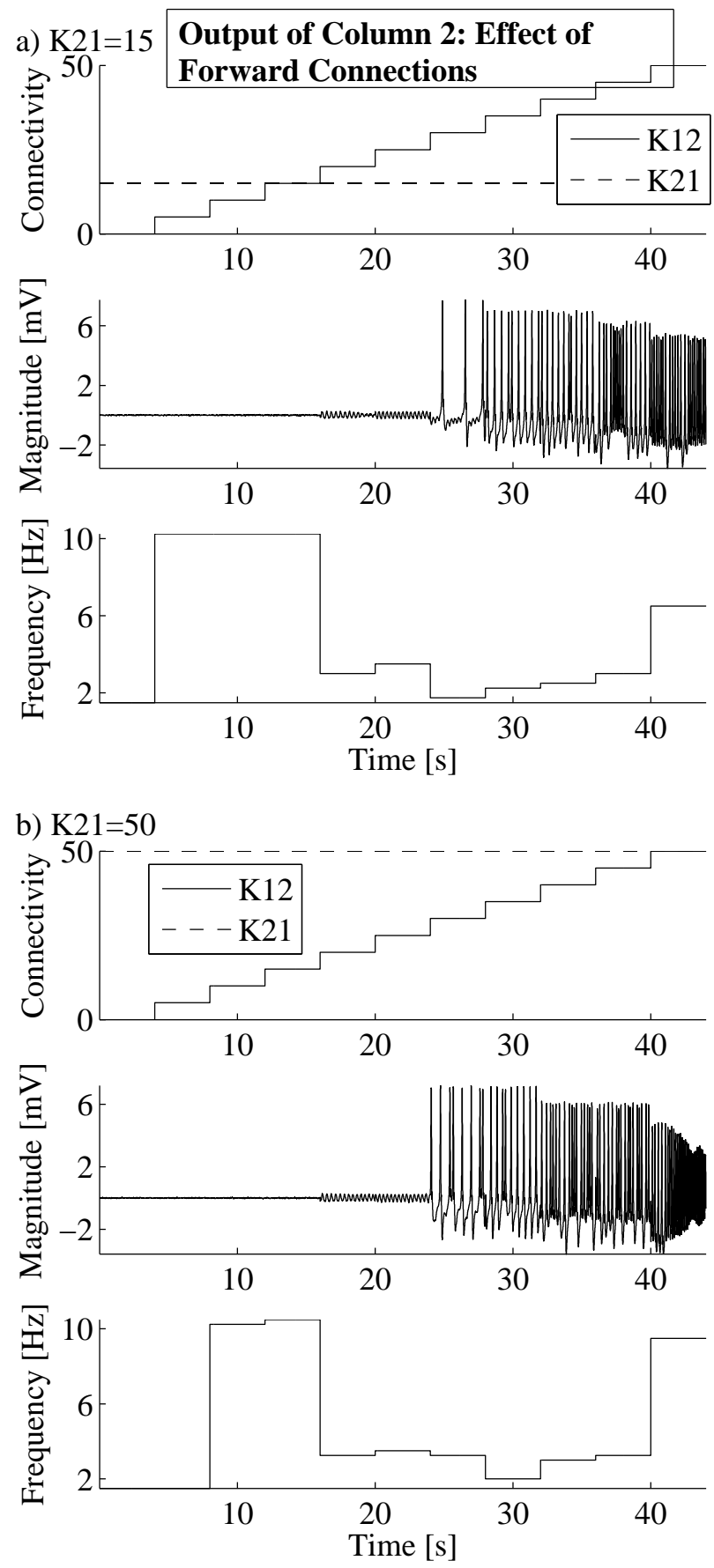

Fig. 7: Output of healthy column 2 when the connectivity strengths between columns 1 and 2 are altered. Top: changes in $K_{12}$ and $K_{21}$ that correspond to the output of column 2 below. Middle: magnitude of the signal output of column 2. Bottom: frequency of the signal output of column 2. a) $K_{21}=15, K_{12}$ varied. b) $K_{21}=50, K_{12}$ varied. $K_{1 j}=K_{12}, K_{i 1}=K_{21}$ $(i, j=3, \ldots, 7), K_{i j}=15(i, j \neq 1)$. Simulation parameters - Column $1: A=5.5 \mathrm{mV}, B=16$ $\mathrm{mV}, G=5 \mathrm{mV}$; Column 2: $A=3.3 \mathrm{mV}, B=22 \mathrm{mV}, G=10 \mathrm{mV}$; Columns 3-7: $A=5.5 \mathrm{mV}$, $B=50 \mathrm{mV}, G=20 \mathrm{mV}$. 


\section{Output of Column 1}
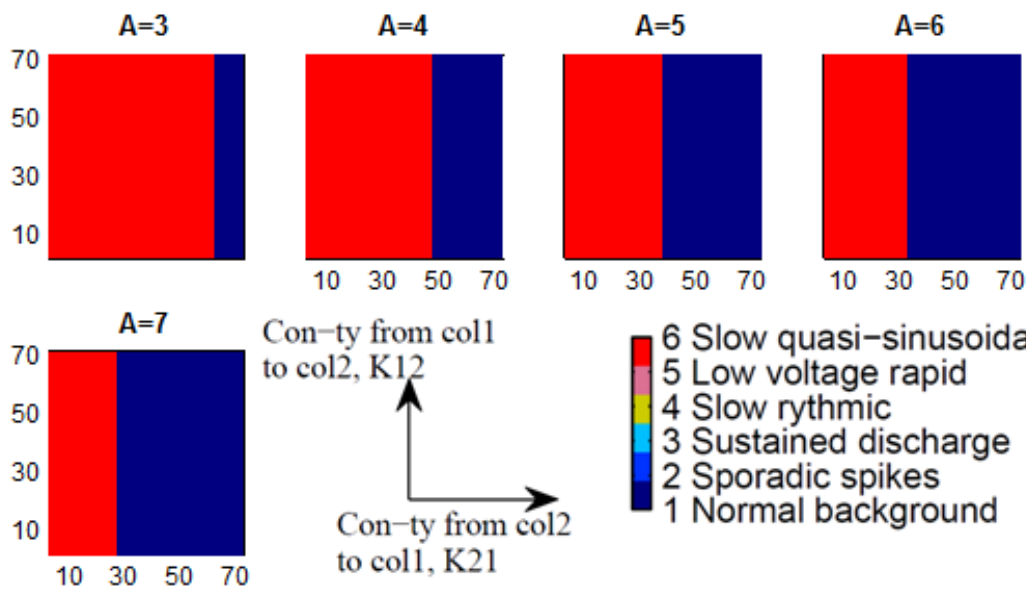

Con-ty from coll to $\operatorname{col} 2, \mathrm{~K} 12$

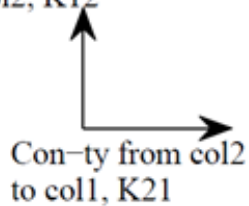

6 Slow quasi-sinusoidal

5 Low voltage rapid

4 Slow rythmic

3 Sustained discharge

2 Sporadic spikes

to col1, K21

Fig. 8: Output of pathological column 1. Dynamics is shown for different connection strengths from the healthy to the pathological column and for different excitatory synaptic gain, $A$, in the healthy column. Simulation parameters - Column 1: $A=5.5 \mathrm{mV}, B=16 \mathrm{mV}, G=5 \mathrm{mV}$; Columns 2-7: $A$ varies, $B=5 \mathrm{mV}, G=5 \mathrm{mV}$. Colorbar corresponds to the activity Types 1-6 illustrated in Fig. 3. 


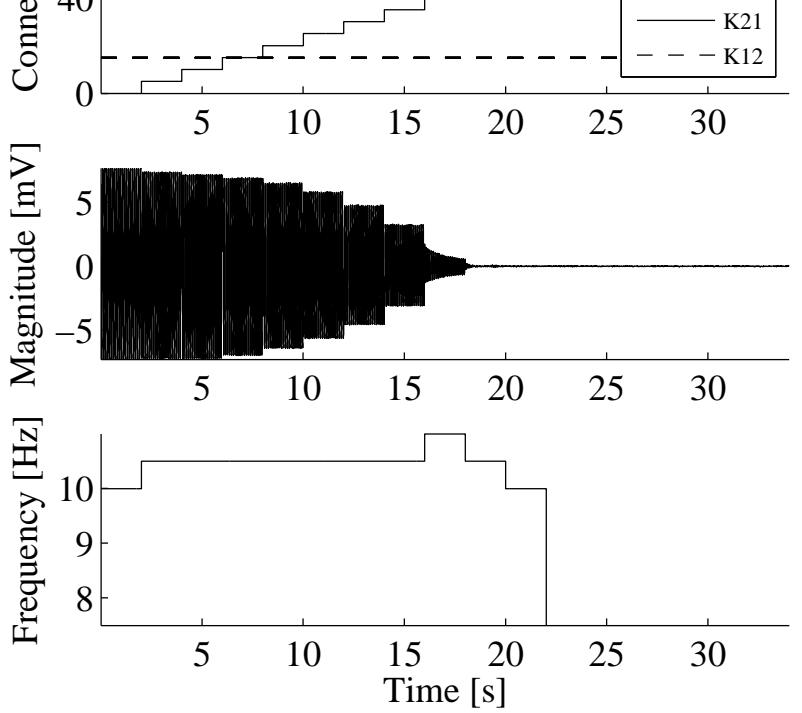

b) $\mathrm{A}=10 \mathrm{mV}, \mathrm{B}=5 \mathrm{mV}$
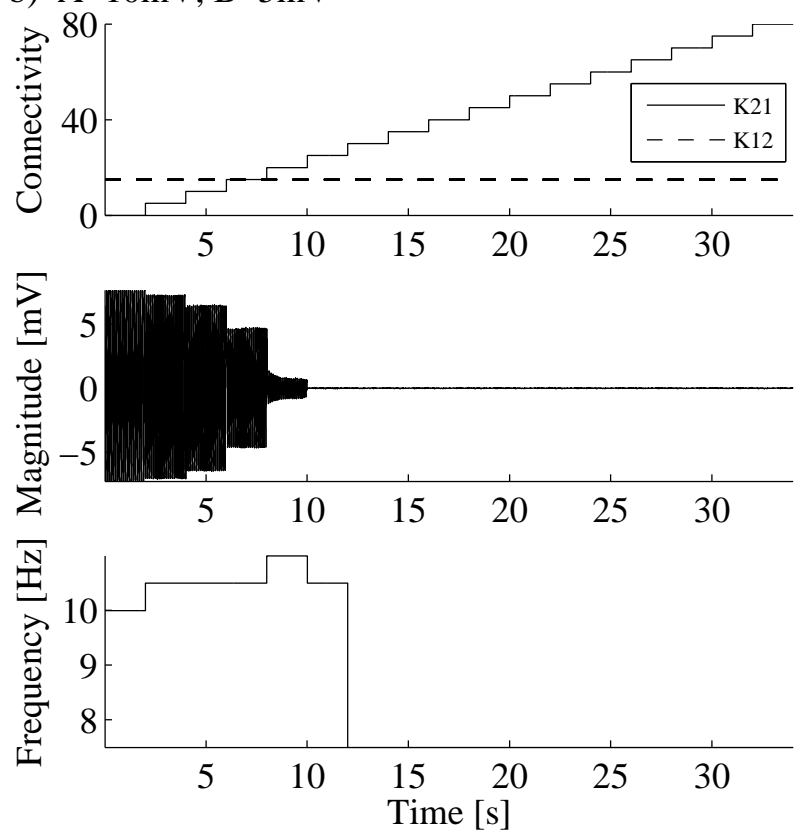

c) $\mathrm{A}=10 \mathrm{mV}, \mathrm{B}=45 \mathrm{mV}$
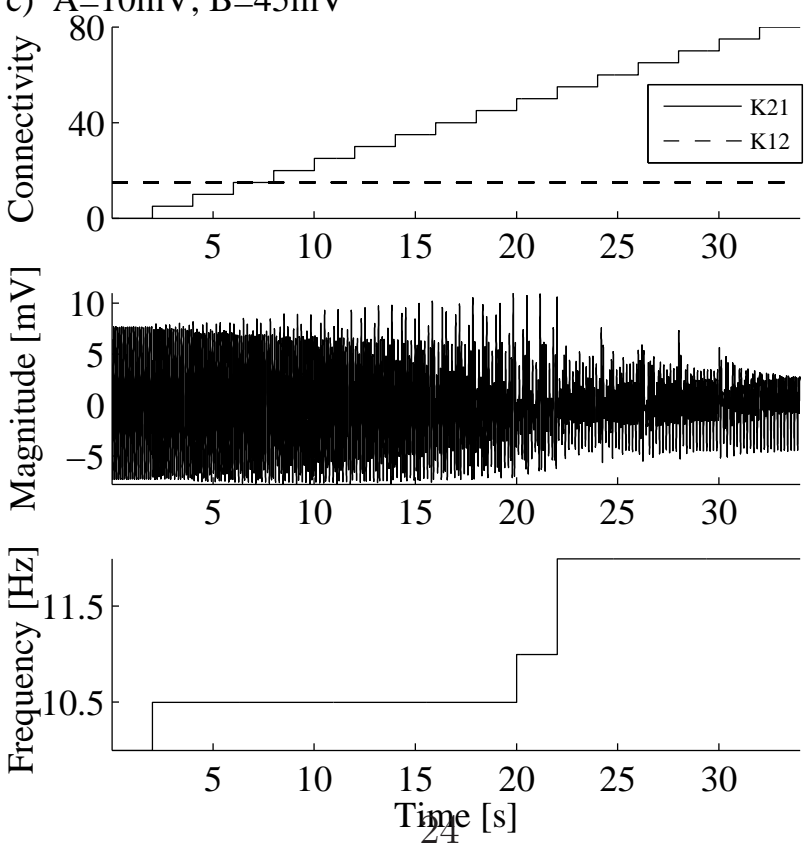

Fig. 9: Output of pathological column 1. Seizure suppression in column 1 when the connectivity strength from neighboring column 2 to pathological column 1, and excitatory and slow inhibitory gains are altered. $K_{12}$ is constant, $K_{21}$ varied. 
a) Connectivity $\mathrm{K} 12=\mathrm{K} 21$, large step in $[0,310]$

0

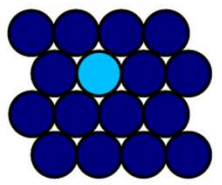

60

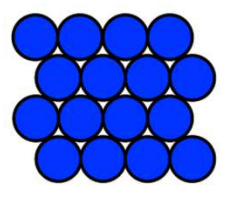

10

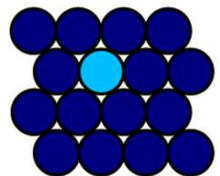

70

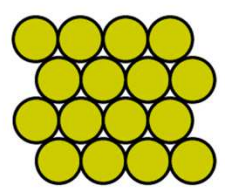

20

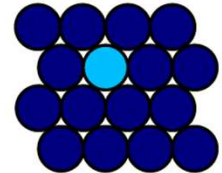

$\cdots$
30

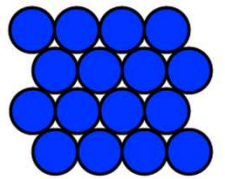

310

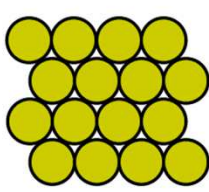

40

50

b) Connectivity K12=K21, small step in [20,30]

20

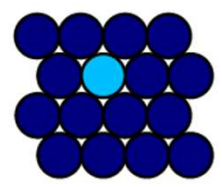

21.92

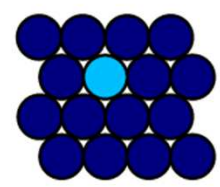

23.84

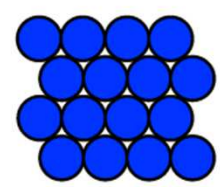

25.76

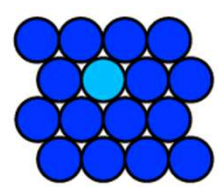

20.32

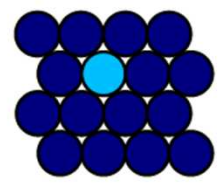

22.24

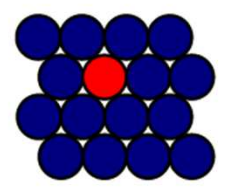

24.16

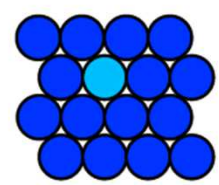

26.08

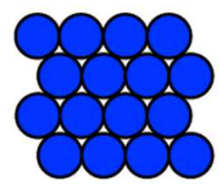

20.64

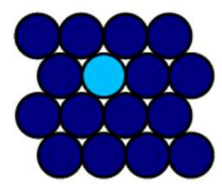

22.56

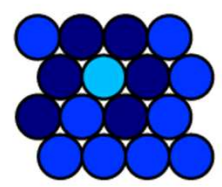

24.48

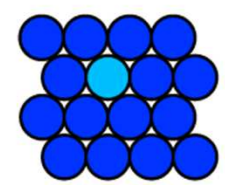

26.4

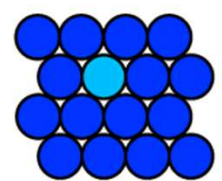

20.96

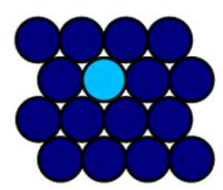

22.88

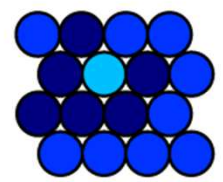

24.8

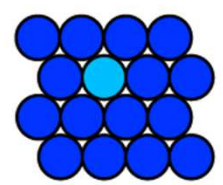

26.72

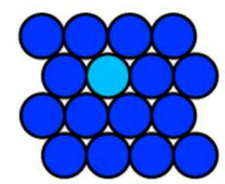

21.28

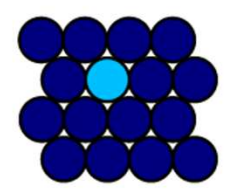

23.2

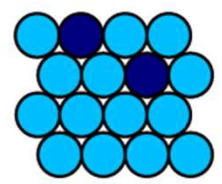

25.12

25.44
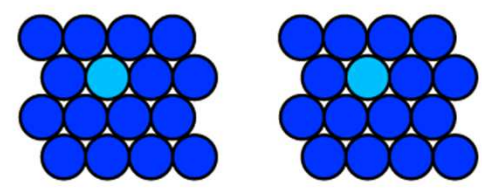

27.04

29.92

21.6

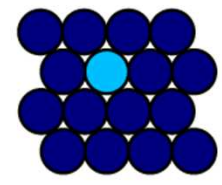

23.52
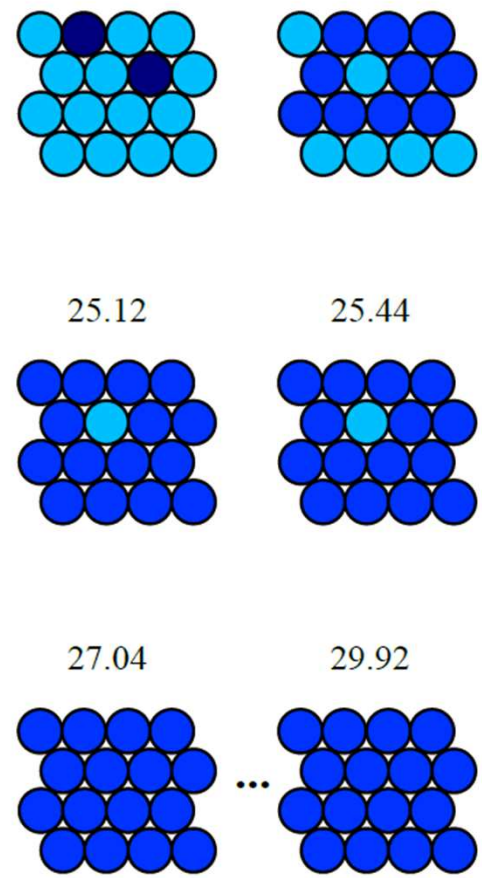

Fig. 10: Seizure propagation for different connectivity in the network. Subplots show spatial dynamics of the network as the connectivity strength, $K_{i j}(\mathrm{i}, \mathrm{j}=1, \ldots, 16)$, is increased a) from 0 to 310 ; and b) in small step from 20 to 30 . Subplots show a network of 16 columns arranged in $4 \times 4$ hexagon. The values for the connectivity constant, $K_{i j}$, used in simulations is shown above each block. $K_{i j}=K j i$ (for all $i, j$ ). Simulation parameters - pathological column (at the intersection of the second row and second columns): $A=6 \mathrm{mV}, B=20 \mathrm{mV}, G=10 \mathrm{mV}$ (Type 3: sustained discharge of spikes); all other columns: $A=3.25 \mathrm{mV}, B=22 \mathrm{mV}, G=18 \mathrm{mV}$ (Type 1: normal background activity when disconnected from the network). Color represent activity type of the network as identified in Figure 3 . 